\title{
BENNETT'S FRACTURE
}

\section{A Method of Treatment with Oblique Traction}

\author{
Olle Spångberg and lars Thorén, Uppsala, Sweden \\ From the Department of Surgery, University of Uppsala
}

The treatment of Bennett's fracture has varied between physical therapy only and open reduction with osteosynthesis (Gedda 1954). An exact reduction is essential for a good result. Gedda (1954) showed that any residual displacement of the joint was always followed by a secondary arthrosis with permanent symptoms.

A new method of treatment by oblique traction was reported in 1955 by Thorén, but at that time only ten cases had been treated and the follow-up period was short. We have now operated on thirty-four cases and present here the follow-up of all the cases and our experiences of the method.

\section{METHOD}

The main characteristic of Bennett's fracture is that a large fragment, comprising almost the whole of the first metacarpal bone, is separated from a small fragment, which remains in its place or is only slightly displaced. In comminuted fractures the smaller fragment is split into several pieces. At reduction the fragments must be

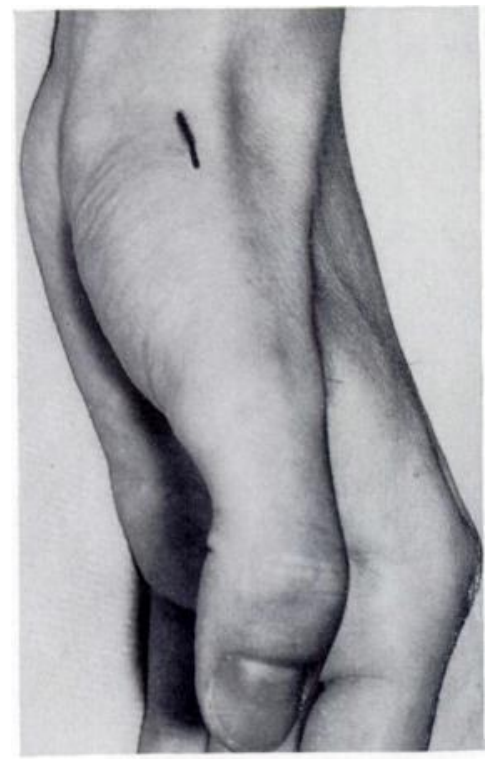

FIG. 1

The incision. apposed and the difficulty is to maintain this position. The larger fragment tends to dislocate in a proximal, dorsal and radial direction due to the pull of abductor pollicis longus and the flexor muscles. Our method resists the tendency to dislocation by traction in a distal, ulnar and palmar direction applied to the base of the first metacarpal bone which reduces the fracture and maintains the alignment.

A longitudinal incision one centimetre long is made over the base of the first metacarpal bone just distal to the insertion of abductor pollicis longus and anterior to the tendon of extensor pollicis brevis (Fig. 1). A Kirschner wire is then drilled through the base of the first metacarpal bone so that it comes out of the thumb web in a distal, palmar and ulnar direction. Thorén (1955), in dissection studies, showed that a wire so inserted is situated between the transverse head of adductor pollicis and the first dorsal interosseous muscle, an area free of nerves, tendons and vessels, so that no important structures are damaged.

The proximal end of the wire is then bent to form a hook which grasps the cortex of the metacarpal (Fig. 2).

By traction on the wire, the fracture can now be reduced and this is sometimes accompanied by a little click. A check radiograph is taken and the method of Billing and Gedda (1952) is best in order to get an exact lateral projection. If a satisfactory position is not obtained, the incision is extended about one centimetre proximally and the joint is opened. There is then no difficulty in reducing the fracture under direct vision and removing any interposed fragments. The reduction is maintained by connecting the Kirschner wire with a rubber 
band to a steel wire frame incorporated in a forearm plaster (Fig. 3). The thumb web must be free from plaster-of-Paris. The exit wound of the Kirschner wire is dressed and a final check radiograph is taken.

The alignment is checked by radiographs taken on the first, third and seventh days after operation and then once a week and the traction modified if necessary. All the finger joints including the metacarpo-phalangeal joint of the thumb are exercised from the first day after operation. Traction is discontinued after five weeks by pushing the wire proximally so that the hook is felt beneath the skin. A small incision is then made under local anaesthesia and the hook removed. The wire is then extracted distally.

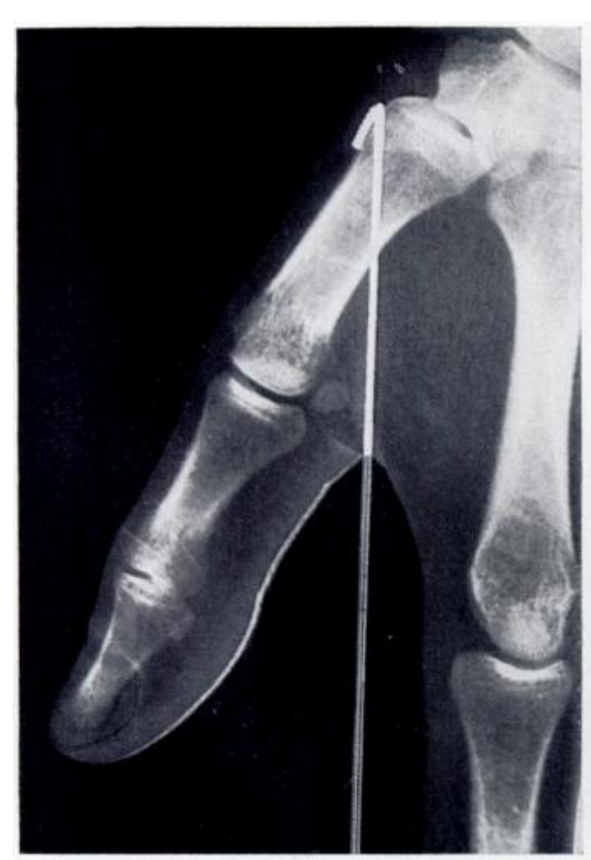

Fig. 2

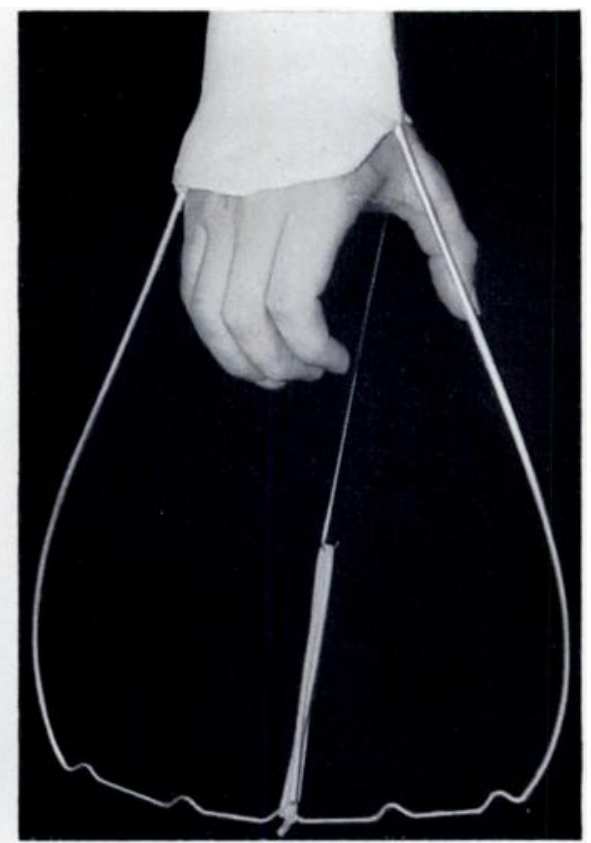

FIG. 3

Figure 2-A Kirschner wire is drilled obliquely through the base of the first metacarpal bone. The proximal end of the wire is bent into a hook. Figure 3-Traction is applied to the wire by rubber bands connected to a steel wire frame incorporated in a forearm plaster.

\section{RESULTS}

The thirty-four cases on which we have operated were those in which accurate closed reduction was impossible. Twenty-seven patients were treated within fifteen days of injury, the remaining seven being treated between twenty-one days and one and a half years after injury. Of the thirty-four cases, twelve were comminuted (Figs. 4 to 6) and twenty-two consisted of a single palmar fragment (Figs. 7 to 9).

All the fractures were caused by indirect violence and none was complicated. There was a transverse fracture of the first metacarpal bone distal to the Bennett's fracture in two cases (Figs. 10 to 12) and in another case the trapezium was fractured.

In three cases a further operation was necessary and these require a more detailed description. In one of these there was a transverse fracture distal to the Bennett's fracture which redislocated three weeks after the reduction. It was not possible for us to supervise the healing process as the patient returned to her home town some distance away. The second case was a neglected fracture first seen one and a half years after injury. At the first operation

VOL. 45 B, No. 4, NOVEMber 1963 


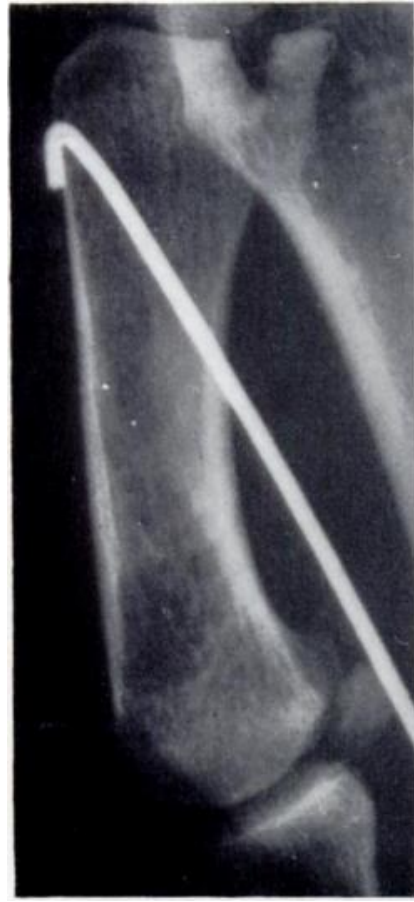

Fig. 4

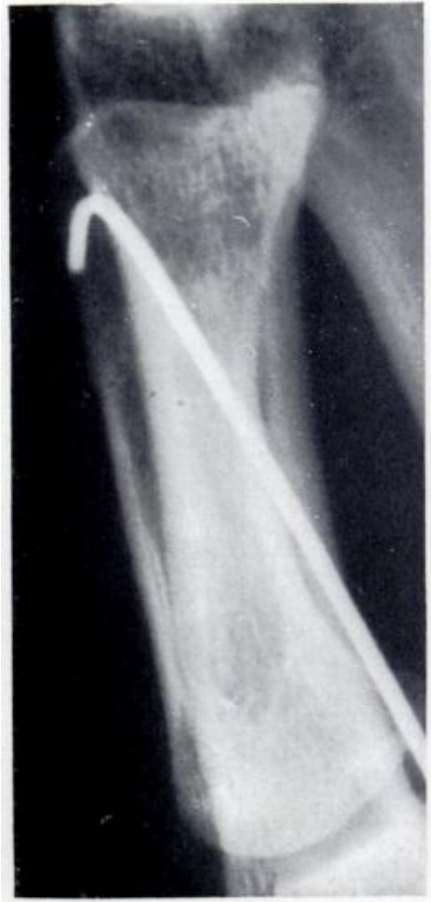

Fig. 5

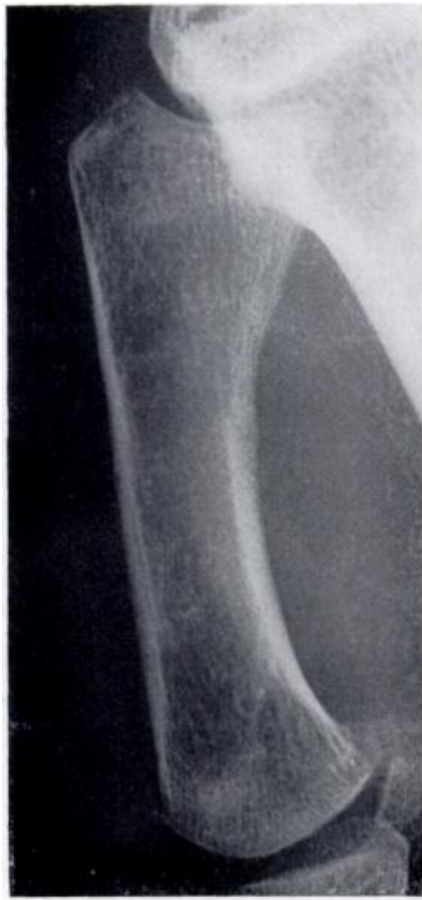

FiG. 6

Figure 4-Bennett's fracture with an intermediate fragment preventing reduction. Figure 5-Accurate reduction after removal of the fragment. Figure 6-The final result.

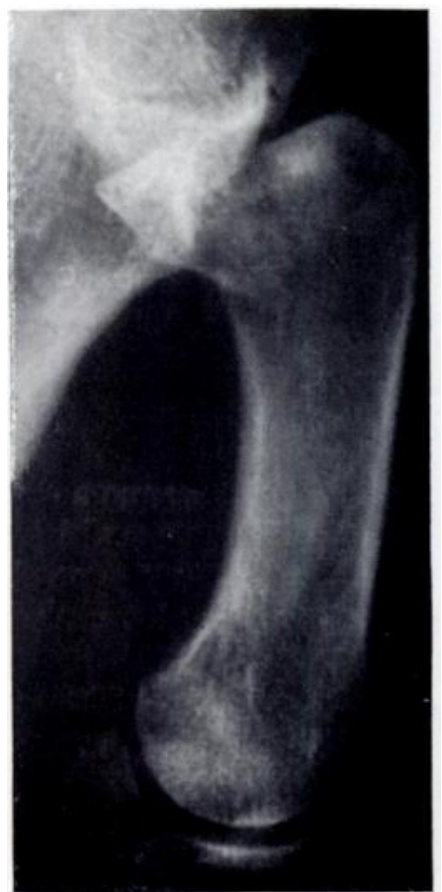

Fig. 7

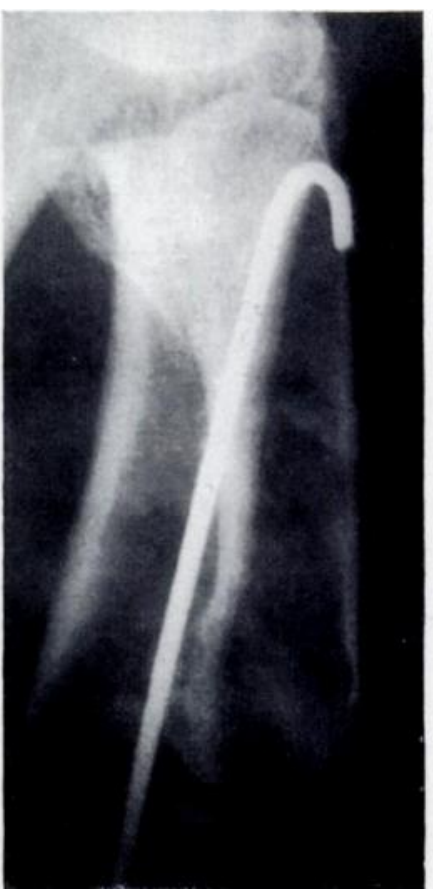

FIG. 8

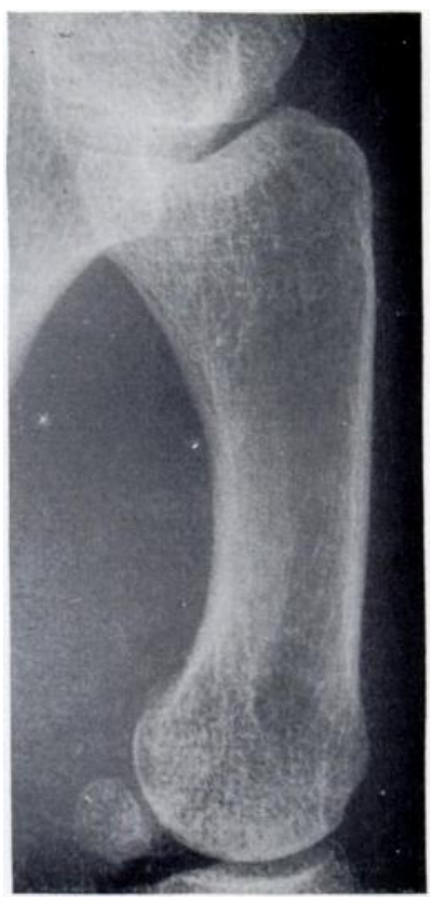

FIG. 9

Figure 7-A common type of Bennett's fracture. Figure 8-After traction has been applied. Figure 9The end result. 
malunion necessitated refracturing the bone after which exact reduction was obtained. When traction was removed after four weeks redislocation occurred. Reapplication of the traction regained exact alignment. In the third case dislocation occurred during traction and could not be improved by increasing the traction. However, open reduction and continued traction were successful.

Arthrotomy was carried out in ten cases. Four of these were recent comminuted fractures and an intermediate fragment was removed in two cases. Arthrotomy was carried out in five of the seven neglected fractures.

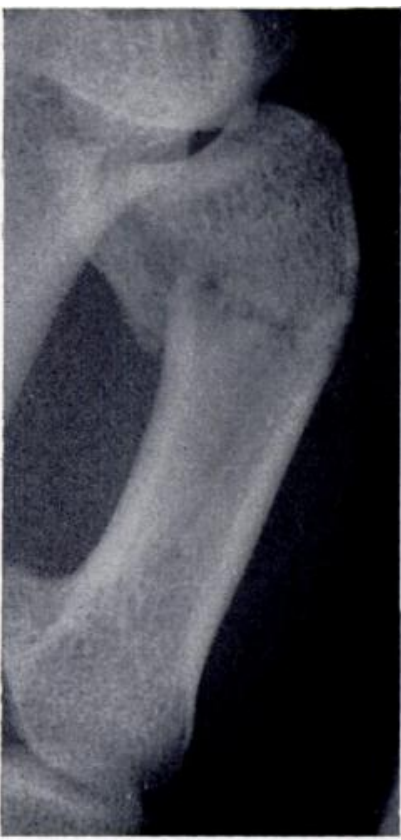

FIG. 10

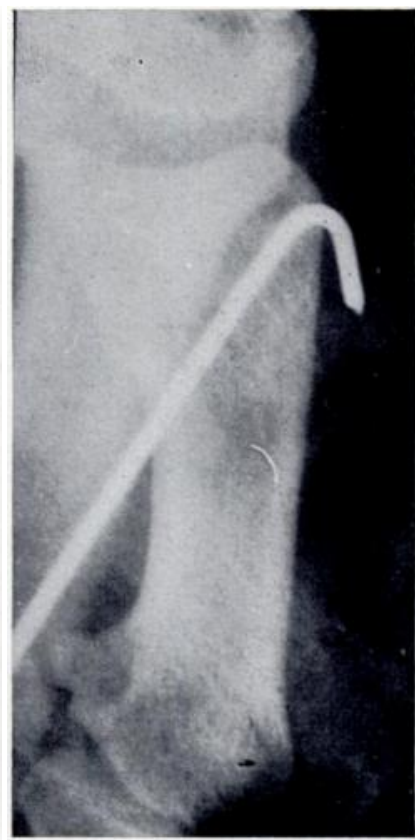

Fig. 11

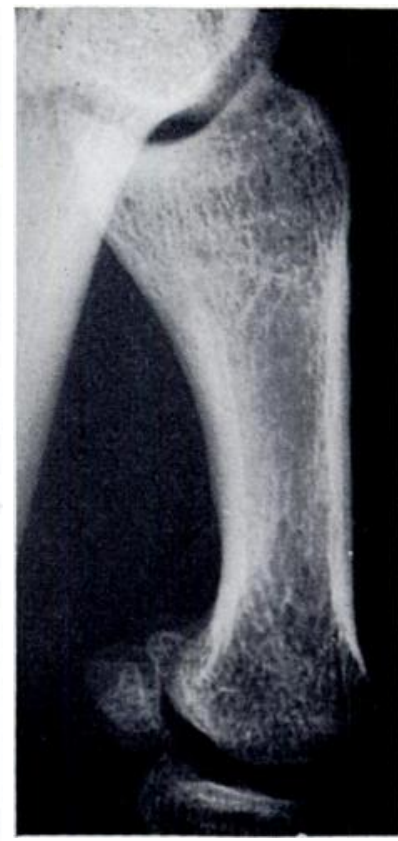

Fig. 12

Figure 10-Bennett's fracture with an additional fracture across the base of the first metacarpal bone. Figure 11-After traction had been applied. Figure 12-The end result.

\section{FOLLOW-UP}

The period of follow-up in all thirty-four patients varied from one year to eight years eight months. Thirty-two patients were symptomless but two complained of some pain at the fracture site especially in cold weather.

There was full movement of all the finger joints in all the patients. In one instance the fracture had united in slight rotation, with the result that the patient was not completely satisfied with the grip between the tip of the index finger and the thumb.

Muscle power was examined with two different types of ergometer (one constructed by Moberg and the other by Silfverskiöld). Impaired power was found in four cases. Radiographs of thirty-two patients showed perfect reduction in twenty-five. In seven the position was not exact. Five of these cases showed a step of less than one millimetre in the basal joint surface of the first metacarpal bone. In one case there was some rotation and in another a bone-like projection had developed on the radial side. Arthrosis had not occurred in any patient.

The average time lost from work after operation was two months. All the patients followed up said that they had no disability.

\section{DISCUSSION}

Undislocated Bennett's fractures do not present a problem and are easy to treat with a plaster cast. Dislocated fractures sometimes present considerable problems. In the first place 
the larger metacarpal fragment must be approximated to the smaller palmar fragment and fixation is a problem. In the second place relatively strong muscle traction causes a dislocation which is difficult to overcome and for a good result accurate reduction must be obtained. The thickness of the articular cartilage on the base of the first metacarpal is two millimetres, so a step in the joint surface radiographically less than one millimetre probably does not

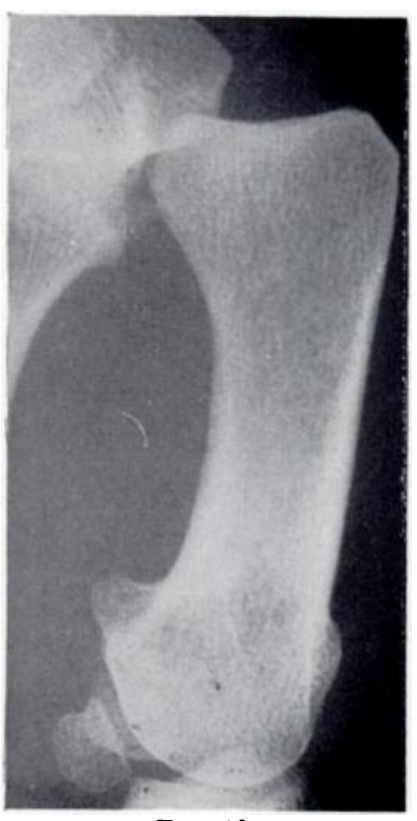

FIG. 13

Dislocation of the first metacarpal bone with a minute palmar fragment. prevent a good result. The number of methods of treatment described suggests that the ideal method has not yet been found. The method described here aims at producing extension in the ideal direction to overcome the tendency to dislocation.

This method is technically easy, no complications have been encountered, and exact open reduction can be achieved when necessary. In our experience, neglected and comminuted fractures often require arthrotomy to obtain accurate reduction.

While the method can be used in all types of Bennett's fracture we wish to stress its particular advantages in: 1) fractures with a very small palmar fragment in which it is difficult to fix the larger fragment to the smaller (Fig. 13); 2) fractures with one or more intermediate fragments which require arthrotomy and removal of the intermediate fragment; 3 ) fractures of the first metacarpal or another adjacent bone when methods which fix the first metacarpal to neighbouring bones cannot be used. It is true that one fracture in this group failed, but this may have been due to insufficient supervision on our part. This method also has advantages in transverse and spiral fractures of the first metacarpal (Thorén 1957).

The great advantage of the method is that finger exercises can be performed throughout treatment.

\section{SUMMARY}

A method of treatment of Bennett's fracture is described. A Kirschner wire is drilled obliquely through the base of the first metacarpal bone and traction is applied in a distal, ulnar and palmar direction in order to counteract the dislocating action of abductor pollicis longus and the flexor muscles.

The advantages of the method are: 1) It is technically easy and practically without complications. No important structures are liable to be damaged. 2) It can be used in those cases where the palmar fragment is very small. 3) It can be used for comminuted fractures. 4) It can be used in neglected cases where malunion has occurred. 5) It can be used when there are other fractures of the first metacarpal or adjacent bones. 6) It allows exercises of all the finger joints during the whole period of treatment.

\section{REFERENCES}

Billing, L., and Gedda, K.-O. (1952): Roentgen Examination of Bennett's Fracture. Acta Radiologica, 38, 471. Gedda, K.-O. (1954): Studies on Bennett's Fracture. Acta Chirurgica Scandinavica, Supplementum 193.

ThORÉN, L. (1955): A New Method of Extension Treatment in Bennett's Fracture. Acta Chirurgica Scandinavica, $110,485$.

Thorén, L. (1957): Basal Fractures of the First Metacarpal Bone. A Method of Treatment by Extension. Acta Orthopaedica Scandinavica, 27, 40. 